\title{
Cronic non-bacterial osteomyelitis (CNO) in a cohort of pediatric patients: clinical, biological and radiological response to treatment with Anakinra
}

\author{
M Pardeo*, D Pires Marafon, V Messia, R Nicolai, C Bracaglia, F de Benedetti, A Insalaco \\ From 8th International Congress of Familial Mediterranean Fever and Systemic Autoinflammatory Diseases \\ Dresden, Germany. 30 September - 3 October 2015
}

\section{Introduction}

Chronic nonbacterial osteomyelitis $(\mathrm{CNO})$ is the most common autoinflammatory bone disorder in childhood (1). Diagnostic information is provided by TC-99 bone scintigraphy (BS) and/or whole body MRI. Non-steroidal anti-inflammatory drugs (NSAIDs), glucocorticoids, bisphosphonates and tumour necrosis factor inhibitors have been used until now with variable response (2).

\section{Objectives}

To describe clinical, biological and radiological response to treatment with anakinra in patients with $\mathrm{CNO}$ refractory to NSAIDs and bisphosphonates.

\section{Materials and methods}

Seven patients ( 4 females and 3 males) with refractory $\mathrm{CNO}$ were treated with anakinra for at least 6 months in our institution. Response to treatment was evaluated assessing clinical manifestations (pain, local swelling, functional impairment), laboratory findings (C-reactive protein (CRP)), erythrocyte sedimentation rate (ESR)) and serum amyloid A level (SAA))) and number of bone lesions on TC-99 BS at the start of treatment and at 6 months.

\section{Results}

The median age at diagnosis and before starting anakinra was 9.7 years (IQR 7.8-14.7) and 13.3 years (IQR 8.0-15.9) respectively. All were treated with NSAIDs and bisphosphonates as first-line therapy. Glucocorticoid therapy was required in one patients with concomitant recurrent fever

\footnotetext{
Bambino Gesù Children Hospital, Pediatric Medicine-Rheumatology, Rome, Italy
} 